\title{
A New Biobjective Probabilistic Risk-Based Wind-Thermal Unit Commitment Using Heuristic Techniques
}

DOI:

10.1109/TII.2016.2616109

\section{Document Version}

Accepted author manuscript

Link to publication record in Manchester Research Explorer

\section{Citation for published version (APA):}

Bavafa, F., Niknam, T., Azizipanah-Abarghooee, R., \& Terzija, V. (2017). A New Biobjective Probabilistic RiskBased Wind-Thermal Unit Commitment Using Heuristic Techniques. IEEE Transactions on Industrial Informatics, 13(1), 115-124. [7587427]. https://doi.org/10.1109/TII.2016.2616109

\section{Published in:}

IEEE Transactions on Industrial Informatics

\section{Citing this paper}

Please note that where the full-text provided on Manchester Research Explorer is the Author Accepted Manuscript or Proof version this may differ from the final Published version. If citing, it is advised that you check and use the publisher's definitive version.

\section{General rights}

Copyright and moral rights for the publications made accessible in the Research Explorer are retained by the authors and/or other copyright owners and it is a condition of accessing publications that users recognise and abide by the legal requirements associated with these rights.

\section{Takedown policy}

If you believe that this document breaches copyright please refer to the University of Manchester's Takedown Procedures [http://man.ac.uk/04Y6Bo] or contact uml.scholarlycommunications@manchester.ac.uk providing relevant details, so we can investigate your claim.

\section{OPEN ACCESS}




\title{
A New Bi-Objective Probabilistic Risk Based Wind-Thermal Unit Commitment Using Heuristic Techniques
}

\author{
Farhad Bavafa, Taher Niknam, Member, IEEE, \\ Rasoul Azizipanah-Abarghooee, Student Member, IEEE, and Vladimir Terzija, Fellow, IEEE
}

\begin{abstract}
Large penetration of wind generating units in power systems necessitates a flexible unit commitment tool to handle the intermittent nature of these units as well as demand. Moreover, power system operators face not only the risks of wind power curtailments, but also probable unit outages. Therefore, assessing a trade-off between operational costs and such risks is very important. In the proposed approach, the probability of the residual demand falling within the up-and-down spinning reserve imposed by $n-1$ security criterion is converted into a risk index. A new bi-objective probabilistic risk/cost based unit commitment model is proposed to simultaneously minimize both the operational costs and risk. The novel formulation presented provides a new power re-dispatch process to satisfy up-and-down ramp rate constraints. A new operational cycles based unit commitment algorithm is developed. The approach profits from a new non-dominated sorting backtracking search optimization algorithm for extracting the Pareto optimal set. The proposed approach is shown to provide superior results when applied to two test systems: a) 10-unit and b) IEEE 118-bus, 54-unit system.
\end{abstract}

Index Terms - Backtracking search algorithm, Bi-objective optimization, Operation risk, Unit commitment, Wind power penetration.

\section{NOMENCLATURE}

\section{Indices}

$d \quad$ Operation cycles $\in(1, \ldots, n d)$.

$h \quad$ Wind power discrete levels $\in(1, \ldots, H)$.

$i, k \quad$ Generator units $\in(1, \ldots, N)$.

$l \quad$ Demand discrete levels $\in(1, \ldots, L)$.

$m \quad$ Residual demand (RD) discrete levels $\in(1, \ldots, M)$.

$p z \quad$ Prohibited operating zone indices $\in(1, \ldots, N Z)$.

$t \quad$ Hourly scheduling intervals $\in(1, \ldots, T)$.

$z \quad$ Pareto front $\in(1, \ldots, K)$.

\section{Parameters}

$a_{i}, b_{i}, c_{i}, d_{i}, e_{i}$ Cost coefficients of unit $i$.

Manuscript received September 11, 2015; revised May 20, 2016; accepted August 12, 2016.

Copyright (c) 2009 IEEE. Personal use of this material is permitted. However, permission to use this material for any other purposes must be obtained from the IEEE by sending a request to pubs-permissions@ieee.org.

F. Bavafa, and T. Niknam are with Department of Electrical and Electronic Engineering, Shiraz University of Technology, Shiraz, Iran (e-mail: f.bavafa@sutech.ac.ir and niknam@sutech.ac.ir).

R. Azizipanah-Abarghooee is with the Department of Electrical and Electronic Engineering, Shiraz University of Technology, Shiraz, Iran (e-mail: r.azizipanah@sutech.ac.ir). He is also a Research Assistant at the School of Electrical and Electronic Engineering, University of Manchester, Manchester M13 9PL, U.K. (e-mail: rasoul.azizipanahabarghooee@postgrad.manchester.ac.uk).

V. Terzija is with the School of Electrical and Electronic Engineering, University of Manchester, Manchester M13 9PL, U.K. (e-mail: terzija@ieee.org).

\author{
$D_{l, t} \quad$ Discrete realization of demand at time $t$ (MW). \\ $n d_{i} \quad$ Number of operation cycles for unit $i$. \\ $p r_{l, t}^{D} \quad$ Probability of demand being equal to $D_{l, t}$ at time $t$. \\ $R D_{m, t} \quad$ Discrete realization of RD at time $t$ (MW). \\ $\hat{P}_{t}^{w} \quad$ Forecasted value of wind power at time $t(\mathrm{MW})$. \\ $P_{h, t}^{w} \quad$ Discrete realization of wind power at time $t(\mathrm{MW})$. \\ $p r_{h, t}^{w} \quad$ Probability of wind power being equal to $P_{h, t}^{w}$. \\ without wind power curtailment. \\ $p r_{k}^{\text {out }} \quad$ Outage probability of unit $k$. \\ $P_{i}^{\max } \quad$ Minimum power output of unit $i(\mathrm{MW})$. \\ $P_{i}^{\min } \quad$ Maximum power output of unit $i$ (MW). \\ $P_{i, p z}^{L}, P_{i, p z}^{U} \quad$ Respective lower/upper bounds of $p z$ th prohibited \\ operating zone for unit $i$. \\ $r_{i} \quad$ Maximum sustained ramping rate of unit $i(\mathrm{MW} / \mathrm{min})$. \\ $R_{i}^{u p} \quad$ Ramp-up rate limit of unit $i(\mathrm{MW} / \mathrm{h})$. \\ $R_{i}^{d n} \quad$ Ramp-down rate limit of unit $i(\mathrm{MW} / \mathrm{h})$. \\ Shot $_{i} \quad$ Hot start-up cost of unit $i(\$)$. \\ Scold $_{i} \quad$ Cold start-up cost of unit $i(\$)$. \\ $S C_{i} \quad$ Shut-down cost of unit $i(\$)$. \\ $T_{i}^{O N} \quad$ Minimum up-time of unit $i(\mathrm{~h})$. \\ $T_{i}^{\text {OFF }} \quad$ Minimum down-time of unit $i(\mathrm{~h})$. \\ $T_{i}^{\text {cold }} \quad$ Cold start-up time of unit $i(\mathrm{~h})$. \\ $\Delta \quad$ Discretization step of power (MW).
}

Variables

- Random Variables at Time $t$

$D_{t} \quad$ Demand (MW).

$P_{t}^{w} \quad$ Wind power (MW).

$R D_{t} \quad\left(=D_{t}-P_{t}^{w}\right)$ Residual demand (MW).

- Continuous Variables at Time $t$

$P_{i, t} \quad$ Scheduled power output of unit $i(\mathrm{MW})$.

$P_{C u r, t}^{w} \quad$ Scheduled value of wind power beyond that all the realizations of wind power will be curtailed (MW).

$\overline{p r}_{h, t}^{w} \quad$ Probability of wind power being equal to $P_{h, t}^{w}$ when wind is curtailed.

$p r_{m, t}^{R D} \quad$ Probability of RD being equal to $R D_{m, t}$ when wind generation is curtailed.

$R D_{t}^{\text {mean }} \quad$ Scheduled mean value of RD (MW). 
- Binary and Integer Variables at Time $t$

$U_{i, t} \quad$ Operation status of unit $i(1 \sim \mathrm{ON}$ and $0 \sim \mathrm{OFF})$.

$\mathrm{Cur}_{t} \quad$ Wind power curtailment level $\in(1, \ldots, H)$.

$z_{m, t} \quad$ Probabilistic security auxiliary variable.

$c_{i, d} \quad$ Probabilistic security auxiliary variable.

Vectors

$\mathbf{C}_{i} \quad$ Operation cycle's starting time vector of unit $i$.

$\mathbf{X} \quad$ Vector of all decision variables.

$\mathbf{X}_{\text {int }} \quad$ Vector of integer decision variables.

$\mathbf{X}_{c o n} \quad$ Vector of continuous decision variables.

$\mathbf{X}_{m} \quad$ Candidate solution in BSA.

$\mathrm{Fr}_{z} \quad$ Pareto front $z$.

Fr Set of Pareto fronts.

Functions

$F_{1}(\mathbf{X}) \quad$ Total operation cost at the entire scheduling horizon (\$).

$F_{2}(\mathbf{X}) \quad$ Operation risk at the entire scheduling horizon.

$\mathrm{Prob}_{t} \quad$ Probability of the RD prediction error at time $t$ remaining within the up and down system reserve capability.

$f_{i, t} \quad$ Generation cost of unit $i$ at time $t$ (\$).

$S U_{i, t} \quad$ Start-up cost of unit $i$ at time $t(\$)$.

$S D_{i, t} \quad$ Shut-down cost of unit $i$ at time $t(\$)$.

$\bar{P}_{i, t} \quad$ Upper limit of the $i^{\text {th }}$ unit output power at time $t$, (MW).

$P_{i, t} \quad$ Lower limit of the $i^{\text {th }}$ unit output power at time $t$, (MW).

$S R_{t}^{u p} \quad$ System up spinning reserve at time $t(\mathrm{MW})$.

$S R_{t}^{d n} \quad$ System down spinning reserve at time $t$ (MW).

Ceil Round function toward positive infinity.

rand Uniformly distributed random number generator in interval $[0,1]$.

$\operatorname{randi}(T, n d)$ Random integer generator in the form of 1-by-nd vector drawn from the discrete uniform distribution on 1:T.

\section{INTRODUCTION}

$\mathrm{W}_{\mathrm{in}}^{\mathrm{i}}$ IND POWER GENERATORS play one of the key role in combatting climate change. The level of their integration into existing power networks is significantly increased. However, inherent variability of wind power alongside demand uncertainty calls for new decision support tools. Since flexibility of conventional thermal units (TUs) is restricted by factors like valve-point effect, the prohibited operating zone, up/down ramp rate, minimum on/off time, as well as up/down spinning reserve (SR) capacity constraints, there is an increasing need for turning on more and more TUs and consideration of optimal wind power curtailments (WPCs). Moreover, the management of operation risk (OR) associated with the unpredictability of wind power and demand as well as probable unit outages is one of the major challenges faced by the system operator [1].

The proposed risk/cost-based unit commitment (RCUC) problem not only takes into account power system's risk and cost, but considers the optimal delivery plan of wind power generators and TUs as well as best commitments of TUs, optimal WPC and also efficient SR accounting for uncertainties associated with the residual demand (RD) and unit outages. System operator needs to have sufficient up-anddown SR to properly manage power systems' uncertainties and contingencies. To the best of our knowledge, the previous works in the area of unit commitment (UC) are related to solution methodologies for UC [2]-[6], risk-constrained UC and modeling related uncertainties [6]-[9], and scheduling of sufficient reserve [10]-[12].

A hybrid genetic algorithm and differential evolution, particle swarm optimization, Matroid theory, mixed integer linear programming and enterprise control techniques are developed in [2]-[6] to solve different UC problems.

As proposed in [1], spinning reserve is set to a fixed percentage of demand to protect power systems against deterministic rules and static criteria such as loss of fixed ratio of demand and outage of largest unit. The first work to consider some predefined spinning reserve requirements for every unit of time in the scheduling horizon taking into account wind power uncertainty, demand and the ramp rate limits of TUs is presented in [7]. In [8], the expected-energynot-served and expected WPC are considered as the risks. In [9], both the expected-energy-not-served and value-of-loss-ofload are considered in the cost function and constraints.

A stochastic programming-based scheduling method (stochastic UC (SUC)) is proposed in [10] to ensure sufficient SR in the UC problem for management of uncertainties. In this method, a large number of scenarios is initially generated and then reduced to reach the desired computation time. However, there is a chance of losing information during scenario reduction. In [11], a risk-based UC formulation is presented to incorporate the risks of the expected-energy-not-served, WPC and branch overflow affected by wind power uncertainty, while neglecting the re-dispatch of conventional TUs for wind power forecasting errors in power flow calculation process. In [12], a chance-constrained programming for handling costbased UC and modeling the day-ahead probability distribution function (PDF) of RD comprising of the WPC influence is presented.

The main contributions of this paper are as follows:

i) Impacts of high wind power penetrations (WPPs) on the RCUC problem considering both the operational cost and risk are modeled. [1], [8], [9], and [11] mainly focus on how to tackle with the uncertainties considering a predefined risk level, which means the risk cannot be adjusted. Different from literatures that explore the SUC operation solutions for minimizing the operational cost and increasing the committed capacity to attain the target risk probability, the suggested model minimizes the operation cost and risk, simultaneously. In this regard, the PDF of the RD is determined based on the estimated PDF of the demand and wind power and optimal WPCs. Then, the probability of the RD falling within the up and down SR imposed by $n-1$ security criterion is determined. Thus, the proposed framework tries to minimize the operational cost and to maximize its probability (this is equivalent to minimize the OR). Furthermore, the cost objective of the proposed problem is different from those proposed in other papers, so that the valve-point effect and prohibited operating zone are also added.

ii) The presence of binary variables in the decision variables' matrix of the UC problem causes negative 
influences on solution qualities of the optimization technique. To tackle all these limitations, in this paper an efficient operation cycle strategy based on minimum up- and down-time is suggested. It can characterize the on/off status of units and optimally handle the UC control variables. In this method, the binary variables are replaced by integer operation cycles without previous restrictions in [13]. This leads to a substantial drop down of the number of decision variables and better numerical results.

iii) To avoid changes in the TUs' status and the use of any penalty factors for satisfying the up/down ramp rate limits, which negatively impacts on the number of feasible and practicable solutions as well as the convergence speed, a new re-dispatch process is proposed to correct the TU's output power. The main benefit of this unique strategy is that it can provide feasible and reliable power outputs with a reasonable computational time.

iv) The main shortages of the previous UC-based optimization works done on this subject are: a) The way they treated the optimization problem from the technical viewpoint and b) The objective functions they have considered (not being techno-economically chosen). Most of the approaches presented in [7]-[12] are only focused on single objective optimization problem in their evaluations [7]-[12]. Additionally, the developed frameworks in [1]-[6], [13] have not delved into the probabilistic treatment of the uncertain and stochastic factors existing in the decision making under study. To this end, a new meta-heuristic bi-objective algorithm, named non-dominated sorting backtracking search optimization (NSBSO), is modeled to cope with the biobjective optimization problem. By combining the new nondominated sorting technique [14] and the backtracking search algorithm (BSA) [15], the NSBSO solves the RCUC problem under uncertainty and optimizes the OR simultaneously. Hence, the flexibility of RCUC is further improved using the NSBSO. The proposed algorithm also utilizes a stronger modified version of the conventional algorithms such as differential evolution. By achieving a well-distributed Pareto frontier (non-dominated solutions) and choosing the best compromise, one can help the system operator to have a better view over the values of operational cost versus the risk level. To verify the suitability of the presented approach, it is applied to two 10-unit and IEEE 118-bus with 54-unit test systems.

\section{PROBLEM FoRMULATION}

\section{A. Operational Cost Objective Function}

The objective function of UC as a well-known problem in power system operation is commonly modeled as (1a) which includes the energy production costs presented in (1b) and start-up/shut-down costs provided in (1c) and (1d) [2]-[6], [13], [16].

$$
\begin{aligned}
& \operatorname{Min}_{1}(\mathbf{X})=\sum_{t} \sum_{i}\left(f_{i, t}+S U_{i, t}+S D_{i, t}\right) \\
& \mathbf{X}=\left[\mathbf{X}_{1}, \mathbf{X}_{2}, \ldots, \mathbf{X}_{T}\right] \text { and } \mathbf{X}_{t}=\left[\begin{array}{lllll}
U_{1, t} & \ldots & U_{N, t}, P_{1, t} & \ldots & P_{N, t}
\end{array}\right]^{T}
\end{aligned}
$$
where

$$
\begin{aligned}
& f_{i, t}=U_{i, t}\left(a_{i}\left(P_{i, t}\right)^{2}+b_{i} P_{i, t}+c_{i}+\left|d_{i} \times \sin \left(e_{i} \times\left(P_{i}^{\mathrm{min}}-P_{i, t}\right)\right)\right|\right) \\
& S U_{i, t}=\left\{\begin{array}{ll}
\operatorname{Scold}_{i} \cdot U_{i, t}, & \text { if } \sum_{t^{\prime}=t-T_{i}^{\text {OFF }}-T_{i}^{\text {old }}}^{t-1} U_{i, t}=0 \\
\operatorname{Shot}_{i} \cdot U_{i, t}\left(1-U_{i, t-1}\right), & \text { otherwise. }
\end{array} \forall i ; \forall t\right. \\
& S D_{i, t}=S C_{i} \cdot U_{i, t-1}\left(1-U_{i, t}\right), \quad \forall i, t
\end{aligned}
$$

\section{B. Operational Risk Objective Function}

The previous works implemented different strategies to handle the OR. The OR is incorporated into the formulation as a hard bound constraint in [7]. In some of them, the operational cost is penalized by the cost of risk i.e. cost of expected-energy-not-served or value-of-loss-of-load [8], [9]. In [10], the pre-contingency cost of security constraints is minimized. In [11], the risk of expected-energy-not-served and WPC are modeled based on the PDF of RD and included in both the operational cost and the constraints. These works only model one of the risk constraints, those are, expected-energynot-served, WPC, and contingency constraints. In this study, a probabilistic index is formulated to quantify the OR function.

\section{- $R D$ uncertainty characterization}

In spite of great progresses in forecasting techniques, the exact prediction of wind power is still impossible for scheduling studies. In this case, the WPC can be considered as a technically viable control measure that the system operator can implement. The WPC represents maximum hourly caps (in MW) beyond which any generation of wind power is considered excess and spilled. The WPC decision variable can be employed by the system operator through the pitch angle controlling mechanism. It has three benefits: i) guaranteeing the feasible UC even in the presence of high wind power combined with low demand; ii) decreasing the RD uncertainty; and iii) complying with the desired OR. To model WPC, we firstly convert demand and wind power PDFs to respective $L$ and $H \quad$ discretized levels $D_{l, t} \forall l \in(2, \ldots, L)$ and $P_{h, t}^{w} \forall h \in(2, \ldots, H)$ with probabilities of $p r_{l, t}^{D}$ and $p r_{h, t}^{w}$. Moreover, the discrete realization of $P_{h, t}^{w}, D_{l, t}$, and residual demand $R D_{m, t}$ are selected to have the uniform value of $\Delta$. The discrete levels of RD can be formulated as: $R D_{m, t}=D_{l, t}-P_{h, t}^{w}, \quad m=H+l-h ; t=1, \ldots, T$.

Therefore, the PDF of the RD can be obtained by the convolution of the PDFs of the demand and the postcurtailment wind generation as follows:

$$
\begin{aligned}
& p r_{m, t}^{R D}=\sum_{\forall l, h: m=H-h+l} p r_{l, t}^{D} \overline{p r}_{h, t}^{w}, \quad \forall m, t ; \\
& \overline{p r}_{h, t}^{w}= \begin{cases}p r_{h, t}^{w}, & \text { if } P_{h, t}^{w}<P_{C u r_{r}, t}^{w} \\
\sum_{j \geq C u_{t}} p r_{j, t}^{w}, & \text { if } P_{h, t}^{w}=P_{C u_{r}, t}^{w}, \quad \forall h, j, t . \\
0, & \text { if } P_{h, t}^{w}>P_{C u r_{r}, t}^{w}\end{cases}
\end{aligned}
$$

The effect of wind curtailment on discretized wind power PDF is described in (2c). Thus, the expected value of the RD is calculated in the following form:

$R D_{t}^{\text {mean }}=\sum_{m} p r_{m, t}^{R D} R D_{m, t}$ 

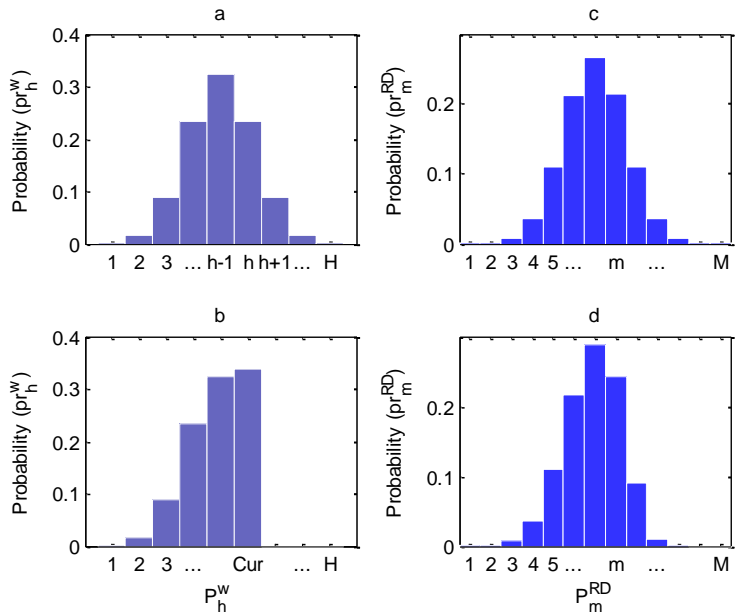

Fig. 1. (a): Original wind power PDF; (b): Wind power PDF after curtailment beyond $\mathrm{Cur}$; (c): Original RD PDF; and (d): RD PDF after curtailment in Cur.

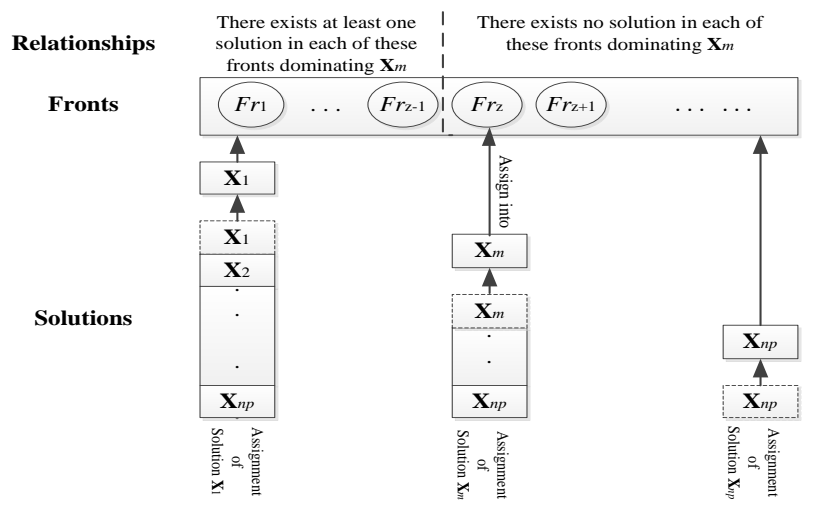

Fig. 2. The whole procedure of the non-dominated sorting method including the solutions assignment and relationships between $\mathbf{X}_{m}$ and the solutions having been assigned to a front.

Algorithm 1: Pseudo-code of the non-dominated sorting method

Input: Population Pop

Output: Set of fronts Fr

Sort $P o p$ in an ascending order of the first objective function. For the solution with the same value of first objective, the second, third and etc. is selected.

for all $\mathbf{X}_{m}$ do

Assign solution $\mathbf{X}_{m}$ into $\mathbf{F r}$ on the basis of Fig. 2.

end for

Return Fr

In order to better illustrate the above procedure, typical wind power PDFs with and without curtailment are depicted in Fig. 1(a) and (b), respectively. The corresponding typical PDFs of RD are also shown in Fig. 1(c) and (d), respectively.

\section{- Operational Risk}

The new binary variable $z_{m, t}$ is defined in such a way that it is 1 , if the RD prediction error falls within available up/down SR. This can be computed as:

$z_{m, t}=\left\{\begin{array}{l}1, \text { if }-S R_{t}^{d n} \leq R D_{m, t}-R D_{t}^{\text {mean }} \leq S R_{t}^{u p} ; \\ 0, \text { otherwise. }\end{array}\right.$

The up/down SRs of the system at each hour are computed according to the following equations:

$$
\begin{array}{ll}
S R_{t}^{u p}=\sum_{i} S R_{i, t}^{u p} \cdot U_{i, t}=\sum_{i} \min \left(P_{i}^{\max }-P_{i, t}, 10 r_{i}\right) U_{i, t}, & \forall t \\
S R_{t}^{d n}=\sum_{i} S R_{i, t}^{d n} \cdot U_{i, t}=\sum_{i} \min \left(P_{i, t}-P_{i}^{\min }, 10 r_{i}\right) U_{i, t}, \quad \forall t .
\end{array}
$$

Now, the probability of the RD forecast error falling within the up/down SR interval can be stated as: $\sum_{m} z_{m, t} p r_{m, t}^{R D}=\operatorname{Prob}\left(-S R_{t}^{d n} \leq R D_{t}-R D_{t}^{\text {mean }} \leq S R_{t}^{u p}\right)$

Thus, the $n-1$ security stochastic criterion is expressed as:

$$
\operatorname{Prob}_{t}=\sum_{k}\left(p r_{k}^{\text {out }} \sum_{m} z_{m, t} p r_{m, t}^{R D}\right) \quad(\forall t)
$$

Using the value of $\operatorname{Prob}_{t}$, the OR is defined as the second objective in the following form:

$\operatorname{Min} \quad F_{2}(\mathbf{X})=\sum_{t=1}^{T}\left(1-\operatorname{Prob}_{t}\right) / T$

It means that the greater the probability of satisfying all the presented systems risks in each hour, the lesser is the OR.

C. Constraints [2]-[13]

$\sum_{i} P_{i, t} U_{i, t}=R D_{t}^{\text {mean }}, \quad \forall t$

$P_{i}^{\min } \leq P_{i, t} \leq P_{i}^{\max }, \quad \forall i, t$

$\left\{P_{i}^{\min } \leq P_{i, t} \leq P_{i, 1}^{L}\right.$

$P_{i, t} \in\left\{P_{i, p z-1}^{U} \leq P_{i, t} \leq P_{i, p z}^{L} \quad p z=1, \ldots, N Z\right.$,

$P_{i, N Z}^{U} \leq P_{i, t} \leq P_{i}^{\max }$,

$\sum_{j=t-T_{i}^{O N}}^{t-1} U_{i, j}=T_{i}^{O N}, \quad$ if $\left(U_{i, t}-U_{i, t-1}=-1\right)$

$\sum_{j=t-T_{i}^{\text {OFF }}}^{t-1} U_{i, j}=0, \quad$ if $\left(U_{i, t}-U_{i, t-1}=+1\right)$

The summation of generated power of all TUs should be equal to RD at each time step which is formulated in (4a). The upper and lower bounds of TUs' generation power output are represented in (4b). In addition, there are some prohibited operating zones in the operation region of the TUs possibly leading to their unsecured operation in prohibited operating zones. The constraint (4c) represents the mentioned subfeasible zones for each TU. Another sever restriction on operation of TUs is the minimum up-time and down-time which are described in (4d) and (4e), respectively. A committed TU is firmly subjected to upward and downward ramping limitations which is pertinent to the operational phase the TU resides. The new re-dispatch process of TUs is proposed in subsection III.D to correct the TU's output power.

\section{SOlution MethodologY}

\section{A. NSBSO Algorithm}

In this paper, the risk/cost-based unit commitment (RCUC) problem is formulated as a bi-objective optimization problem. In contrast to a single-objective optimization problem which has a specific single solution, there is no single solution to optimize all objective functions when the objectives are in conflict with each other, e.g., the lower amount of the operation cost (1a) will result in a higher amount of OR (3f). In this case, different Pareto optimal (also known as nondominated) solutions may exist. It is called Pareto optimal as the solution worsens the condition of at least one objective function while improving others.

Many classical-based optimization techniques are imperfect in providing a Pareto-optimal front, since they can provide one Pareto solution in one run [14]. In addition, they suffer from handling complex bi-objective optimization problems. 


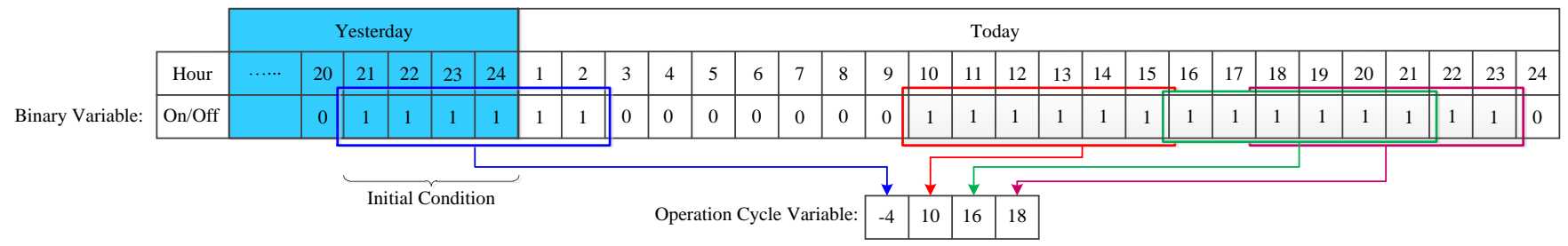

Fig. 3. An example for converting of binary variables to the proposed operating cycle variable for a TU of $T^{O N}=6$.

Due to the ability of meta-heuristic techniques in dealing with the mentioned problems, they are more and more used to manage complex bi-objective optimization problems in the power system area [17] and [18]. In this study, the NSBSO algorithm is used to find a set of Pareto solutions in one single run. In this technique, the non-dominated sorting approach is employed for selection and dominance comparison mechanism [14]. The non-dominated sorting method is computationally intensive, in particular when the population size increases. The detailed description of this method is presented in [14] and can be found in Fig. 2 and Algorithm 1. Afterward, the backtracking search algorithm (BSA) iteratively modifies the position of candidate solutions to enhance the bi-objective optimization problem. The BSA is a population-based metaheuristic algorithm which has been introduced in [15].

The BSA's structure is introduced in five steps of initialization, selection-I, mutation, crossover, and selection-II which are consequently applied during the optimization process of BSA [15].

\section{B. Developed Redefining of On/Off Variables}

In the classical UC formulation, decision variables are classified into two main groups: (i) Binary on/off variables which decide the operation status of units at each time step, and (ii) Continuous variables including the power output of committed units. The variables in the first group are the essential RCUC's variables that affect the convergence speed and global search potential of the introduced NSBSO algorithm. The reader is referred to [19] for more details on the challenges caused by binary variables in optimization problems. To deal with these issues, a new concept of operating or commitment cycles is introduced in this study to convert the binary variables to integer ones with extended range of variation. Accordingly, operational cycle variables can be expressed as follows:

$\left(c_{i, d}=t\right) \approx\left\{\begin{array}{l}\left(U_{i, j}=1, \forall j \mid t \leq j<t+T_{i}^{O N}\right) \text { if } t>0 \\ \left(U_{i, j}=1, \forall j \mid 1 \leq j<t+T_{i}^{O N}\right) \text { otherwise. }\end{array} \forall i, d\right.$

Relation (7) implies that the $d$ th operation period of the $i$ th unit is started from hour $t$ and continued $T_{i}^{O N}$ hours. In order to represent longer period rather than $T_{i}^{O N}$, it is enough to combine two consecutive cycles. The number of required operating cycles of unit $i\left(n d_{i}\right)$ to cover the entire scheduling horizon can be computed as follows:

$n d_{i}=\operatorname{ceil}\left(\frac{T}{T_{i}^{O N}}\right)$

It can be seen that the number of variables in the new form for presenting the commitment status is a function of their minimum up-time hours. It should be mentioned that the constraint $(4 d)$ is satisfied for each unit using (7). To satisfy constraint $(4 \mathrm{e})$, the following restrictions should be satisfied for two consecutive cycles:

$\left(c_{i, d+1}-c_{i, d} \leq T_{i}^{O N}\right) \|\left(c_{i, d+1}-c_{i, d} \geq T_{i}^{O N}+T_{i}^{O F F}\right) \quad \forall i, d$.

where, $c_{i, d+1}-c_{i, d}$ as the difference between the starting times of two consecutive operation cycles $d+1$ and $d$ for the $i$ th TU should be checked through the proposed procedure using (9). Fig. 3 illustrates how binary variables can be converted to operation cycles for a typical TU with $T_{i}^{o N}=6$. According to equation (8), this unit needs four operation cycles. Using these variables results not only in an extreme decrease of RCUC variables' numbers and a subsequence increase in the convergence speed, but also provides an efficient structure that each heuristic algorithm can be applied on these variables. Therefore, the variables' vector $\mathbf{X}$ can be defined as: $\mathbf{X}=\left[\mathbf{X}_{\text {int }}, \mathbf{X}_{c o n}\right] ; \quad\left(c_{i, d}, C u r_{t}\right) \in \mathbf{X}_{\text {int }} \forall i, d, t$ and $P_{i, t} \in \mathbf{X}_{c o n} \forall i, t$.

In a randomly generated initial population, the committed status of any unit is determined as follows:

$\mathbf{C}_{i}=\operatorname{sort}\left(\operatorname{randi}\left(T, n d_{i}\right)\right), \quad \forall i$.

To satisfy constraint (9), the first cycle of each unit is examined. If this cycle violates (9), i.e. $\left(T_{i}^{O N}<c_{i, d+1}-c_{i, d}\right.$ $<T_{i}^{O N}+T_{i}^{\text {OFF }}$ ), the period of next cycle should be corrected as: $c_{i, d+1}=c_{i, d}+T_{i}^{O N}+T_{i}^{O F F} \times \operatorname{round}\left(\frac{c_{i, d+1}-c_{i, d}-T_{i}^{O N}}{T_{i}^{O F F}}\right)$.

Afterwards, the possibility of supplying RD by committed TUs will be checked. For this purpose, the maximum generation of committed units at each hour should be satisfied in the following equation:

$\sum P_{i}^{\max } U_{i, t} \geq R D_{t}^{\text {mean }}, \quad \forall t$.

In the proposed method, in order to supply the required generation with minimum change in the obtained schedules through the optimization process, at first the off-units with the $T_{i}^{O N}$ equal to one hour are turned on. Then, if there is further need for participation of other units, they will be switched on randomly until (12) is satisfied.

\section{Proposed Handling of the Up/Down Ramping Rate Limit}

After settling the hourly TUs' power outputs based on (4a) to $(4 \mathrm{c})$, hours may exist when up/down ramp rate constraints are not satisfied. The modification of TUs' status or penalty functions which negatively impact on the number of feasible and practicable solutions as well as convergence speed have been used in previous literature. Hence, a new method is proposed to correct the TUs' power outputs in the case of ramp rate violations without changing their statuses. 
TABLE I

Results ObTAINED By DifFERENT Methods FOR CASE STUdy 1

\begin{tabular}{c|c|c|c}
\hline Methods & $\begin{array}{c}\text { Beat operation } \\
\text { cost (\$) }\end{array}$ & $\begin{array}{c}\text { Mean } \\
\text { operation } \\
\text { cost (\$) }\end{array}$ & $\begin{array}{c}\text { Mean } \\
\text { Execution } \\
\text { time (s) }\end{array}$ \\
\hline ICGA [13] & NA & 566,404 & 7.4 \\
\hline DP [20] & $565,825.00$ & NA & NA \\
\hline LR [20] & $565,825.00$ & NA & NA \\
\hline GA [20] & $565,825.00$ & NA & 221 \\
\hline SABA [23] & $563,937.70$ & 564,311 & 29 \\
\hline IQEA [24] & 563,942 & 566,170 & 30.54 \\
\hline BSA & $563,937.70$ & 564,227 & 19.4 \\
\hline
\end{tabular}

NA: Not Available

The new constraints are accordingly defined and the new redispatch process is applied for two consecutive hours that the up/down constrains are violated (e.g. $t$ and $t+1$ ). Providing the feasible, reliable as well as optimal power outputs are the main advantages of this method. Note, the proposed method is started from the first time step and continued hour by hour. The proposed re-dispatch algorithm is formulated in (13)-(20) to provide the feasible generation levels:

$\operatorname{Min} \sum_{i}\left(f_{i, t}+f_{i, t+1}\right)$ and or $\operatorname{Min}\left(\left(\left(1-\operatorname{Prob}_{t}\right)+\left(1-\operatorname{Prob}_{t+1}\right)\right) / 2\right)$

Subject to:

$$
\begin{aligned}
& \sum_{i} P_{i, t} U_{i, t}=R D_{t}^{\text {mean }} \\
& \sum_{i} P_{i, t+1} U_{i, t+1}=R D_{t+1}^{\text {mean }} \\
& -R_{i}^{d n} \leq\left(P_{i, t+1}-P_{i, t}\right) \leq R_{i}^{u p}, \quad \forall i . \\
& \underline{P}_{i, t} \leq P_{i, t} \leq \bar{P}_{i, t}, \quad \forall i \\
& \bar{P}_{i, t}=\min \left(P_{i, t-1}+R_{i}^{u p}, P_{i}^{\max }\right) U_{i, t}, \quad \forall i \\
& \underline{P}_{i, t}=\max \left(P_{i, t-1}-R_{i}^{d n}, P_{i}^{\min }\right) U_{i, t}, \quad \forall i \\
& P_{i, t} \in\left\{\begin{array}{c}
P_{i, t} \leq P_{i, t} \leq P_{i, p z^{\prime}}^{L} \\
P_{i, p z^{\prime}}^{U} \leq P_{i, t} \leq P_{i, p z^{\prime}+1}^{L} \quad \forall i, t, t+1 \\
\vdots \\
P_{i, p z^{\prime \prime}}^{U} \leq P_{i, t} \leq \bar{P}_{i, t}
\end{array}\right.
\end{aligned}
$$

In the above mentioned new re-dispatch process, the operation cost or OR of the two hours $t$ and $t+1$ is considered in the single-objective optimization process while both of them should be integrated in the bi-objective optimization problem according to (13). The power balance restrictions of hour $t$ and $t+1$ are referred to in (14) and (15), respectively. The new restriction (16) is defined for TUs so that the difference between their power outputs for two consecutive hours $t$ and $t+1$ will never exceed from their limitations. The acceptable bounds for TUs' power generations considering the up/down ramp rates are updated in the re-dispatching process of RD on the committed TUs. Accordingly, relations (17) to (19) ensure that correction of generation levels of committed TUs at hour $t$ cannot affect the ramp rate constraints at the previous hour $t-1$. The prohibited operating zones strictly pose the restrictions for TUs to participate in load demand regulations. Constraint (20) is the reformulation of (4c) when up/down ramp rate limits are added to the problem. $p z^{\prime}$ and $p z^{\prime \prime}$ are indices of two prohibited operating zones which are located right after and before the $\underline{P}_{i, t}$ and $\bar{P}_{i, t}$, respectively. Note, providing the feasible and reliable power outputs in a short computational time is the main advantage of this unique strategy.

\section{Application of the Proposed Algorithm to the RCUC}

In summary, the procedure of the proposed NSBSO algorithm for solving the RCUC problem will be as follows:

Step 1: Input required data.

Step 2: Generate the initial population, randomly.

Step 3: Calculate the objective functions using (1a) and (3f).

Step 4: Check for non-domination. Constitute the Pareto fronts and determine the Pareto solutions belonging to $F r_{1}$ to $F r_{K}$ using non-dominated sorting method (Algorithm 1). It is clear from Algorithm 1 that if a candidate solution is allocated to a front, it is dominated by at least one in the prior front. It means that $F r_{1}$ is consisted of Pareto solutions.

Step 5: Use the decision-making criterion based on following formulation to select the best compromise solution using the weighting factor and corresponding normalized objective as:

$v_{m}=\frac{\sum_{s=1}^{2} w_{s} \text { norm }_{s}\left(X_{m}^{F r_{1}}\right)}{\sum_{n=1}^{N_{F_{1}}} \sum_{s=1}^{2} w_{s} \text { norm }_{s}\left(X_{z}^{F r_{1}}\right)}$

where, $v_{m}$ is the normalized index for $X_{m}^{F r_{1}} \cdot X_{m}^{F r_{1}}$ and $X_{n}^{F r_{1}}$ are the $m$ th and $n$th members of $F r_{1}$, respectively. $w_{s}$ is the weighting factor assigned to the $s^{\text {th }}$ objective function which is set to 0.5 for both objective functions. norm $_{s}$ is the normalized value of $s$ th objective and $N_{F r_{1}}$ is the size of $F r_{1}$.

Step 6: Modify the position of each particle of population deploying BSA [15].

Step 7: Calculate the objective functions using (1a) and (3f).

Step 8: Go to Step 4 until the current iteration number reaches the pre-specified maximum iteration number.

\section{CASE STUdIES}

\section{A. The First Test System: 10-unit}

Detailed data of this test system can be derived from [20]. It should be noted that different WPPs i.e. $15 \%, 20 \%$ and $25 \%$ are considered for evaluation process. For instance, the hourly wind power generation for the penetration level of $20 \%$ and wind capacity factor $35 \%$ is taken from [21]. The hourly wind power prediction error is assumed to follow a normal PDF with a standard deviation $\left(\sigma_{w}\right)$ [22]. The wind power PDF has disparate standard deviations (Stds) in different hours. For instance, its Std will be increased in proportion of its prediction accuracy reduction. Thus, the $H$ has different values along the day which may change in the range of 7 and 11 . Furthermore, the random behavior of demand forecast errors are modeled by normal PDF with zero mean value and Std equal to $2 \%$ of daily peak demand. As the demand has the fix Std, thus $L$ has a fix value during scheduling horizon. For $\Delta=5 \mathrm{MW}$, the number of discretized levels for demand $(L)$ and wind power realization $(H)$ are equal to 7 and 11 , respectively. It is worth mentioning that the developed software program is written in the commercial FORTRAN Power Station computing environment and applied on a PC laptop with an Intel Pentium IV 2-GHz with 512 MB RAM. 
TABLE II

UC RESULTS USING BSA METHODS

\begin{tabular}{c|ccc}
\hline Algorithms & Operation cost (\$) & $\begin{array}{c}\text { Number of } \\
\text { violated units }\end{array}$ & CPU time (s) \\
\hline BSA\#1 & 512,412 & - & 19.4 \\
BSA\#2 & 512,087 & 2 & 28.3 \\
BSA\#3 & 513,491 & - & 36.7 \\
BSA\#4 & 512,923 & 2 & 55.2 \\
\hline
\end{tabular}

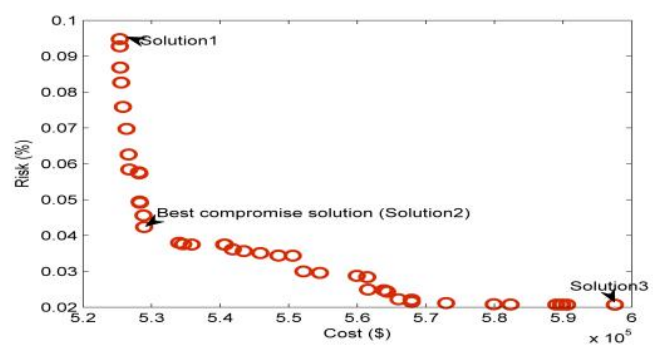

Fig. 4. Pareto-optimal front obtained by proposed NSBSO for case study 3 . The maximum number of iterations and number of particles are set to 100. In order to better illustrate the impacts of wind power plants integrations on the RCUC, numbers of on/off statuses of each unit and their generation levels and WPCs, different WPPs are investigated.

\section{- Case Study 1: Single objective UC without wind power}

The first case is provided for the base 10 TUs without including wind power generators to illustrate the effectiveness of the suggested BSA with respect to other well-known methods such as integer-coded GA (ICGA) [13], dynamic programming (DP) [20], Lagrangian relaxation (LR) [20], GA [20], self-adaptive bat-inspired algorithm (SABA) [23], and intelligent quantum inspired evolutionary algorithm (IQEA) [24]. The results are shown in Table I. It is clear-cut from this table that the proposed method is capable of providing better results. The average CPU time for simulating the proposed algorithm is 19.4 second which is almost lower than other mentioned methods. In a satisfactory time, the best and the average operational costs are also better than other techniques. This indicates that the BSA is more competent while considering several constrains. It is evidently realized from the results that the BSA attains high worthy solutions than other techniques such as SABA [23]. The basic reason behind this event is the superior capability of the BSA along with the proposed redefining binary on/off variables as well as up/down ramping rate handling. Another advantage of this case study is that it presents a meaningful comparison of the proposed approach with traditional DP [20] and LR [20] benchmarks to adequately evaluate its technical and economic benefits.

\section{- Case Study 2: Single objective UC with precise wind power} and demand forecasts' assumption

In this case, the power system's units are planned in the UC problem assuming that wind power and demand predictions are perfect and with zero error. Since it is supposed that there is no error in the forecasting process, the OR will be zero. In this case, the total operational cost is equal to $\$ 512,163.64$ which is approximately $9 \%$ lower than the previous case study. It should be noted that unlike most of the evolutionary algorithms which implements penalty factors to handle the minimum on/off time and ramp rate constraints, this paper applies the proposed heuristic procedures based on the duration of operation and new re-dispatching process.
TABLE III

WIND CURTAILMENT LEVEL OF DIFFERENT SOLUTIONS OF PARETO-OPTIMAL FRONT FOR CASE STUDY 3

\begin{tabular}{ccccccccccccc}
\hline Hours & \multicolumn{10}{c}{$\mathbf{1 - 1 2}$} \\
\hline Solution1 & 7 & 7 & 7 & 7 & 7 & 7 & 7 & 5 & 5 & 5 & 5 & 5 \\
\hline Solution2 & 6 & 6 & 6 & 6 & 6 & 6 & 6 & 4 & 4 & 4 & 4 & 4 \\
\hline Solution3 & 1 & 1 & 1 & 1 & 1 & 1 & 1 & 1 & 1 & 1 & 1 & 1 \\
\hline Hours & & & & & \multicolumn{13}{c}{$\mathbf{1 3 - 2 4}$} & & & & & \\
\hline Solution1 & 5 & 5 & 5 & 5 & 5 & 5 & 5 & 7 & 7 & 7 & 7 & 7 \\
\hline Solution2 & 4 & 4 & 4 & 4 & 4 & 4 & 4 & 6 & 6 & 6 & 6 & 6 \\
\hline Solution3 & 1 & 1 & 1 & 1 & 1 & 3 & 1 & 2 & 1 & 1 & 1 & 1 \\
\hline
\end{tabular}
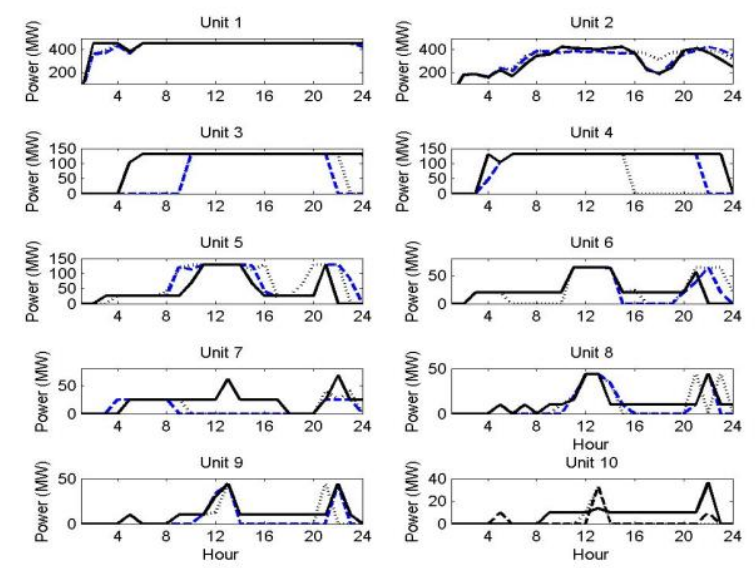

Fig. 5. Best dispatch found by the proposed approach for case study 3: (--): Solution1, (--): Solution2 and (-): Solution3.

The use of penalty factors can only release the violated restrictions and could not eliminate them entirely. Furthermore, the number of feasible solutions as well as convergence speed will be decreased during the optimization procedure. Results of the proposed BSA (BSA\#1), the BSA using the proposed operation cycle approach and the penalty function (BSA\#2), the binary BSA using the proposed up/down ramp rate handling (BSA\#3) and the binary BSA using the penalty function (BSA\#4) are presented in Table II. As can be seen, not only there is no unit with violated ramp rate limits in the case of the BSA\#1, but also the CPU execution time of this algorithm is less than other BSAs. It is also evident that the operational cost of the BSA\#1 is a little more than the BSA\#2. It is because that in BSA\#2, the search space comprises of all the feasible as well as infeasible solutions and it is reasonable to provide better operational cost while violating some of the ramp rate limits. Penalty functions of BSA\#2 negatively impact on the number of feasible and practicable solutions as well as convergence speed. However, its computational time is decreased to $28.3 \mathrm{~s}$ compared to BSA\#4 i.e. $55.2 s$ due to implementation of the proposed operational cycle.

\section{- Case Study 3: Bi-objective RCUC considering wind power and demand forecasts' errors}

In this case, the bi-objective optimization problem is solved using the NSBSO. An efficient Pareto-optimal front including 43 Pareto solutions are provided and shown in Fig. 4, which presents the cost-benefit characteristics of up/down SR consideration to the SO. This large range of non-dominated solutions satisfies the operation requirements in practical power systems. 


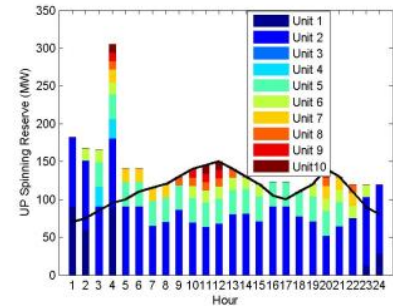

(a1)

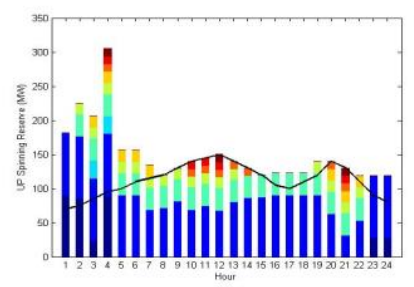

(b1)

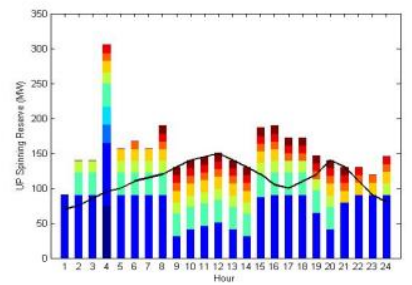

(c1)

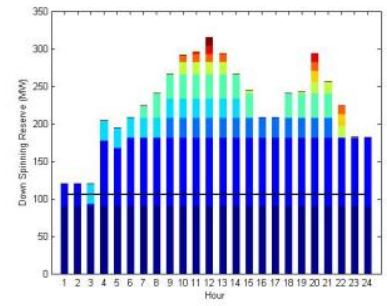

(a2)

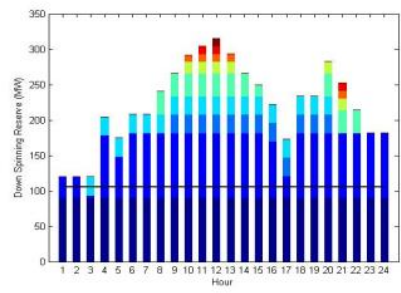

(b2)

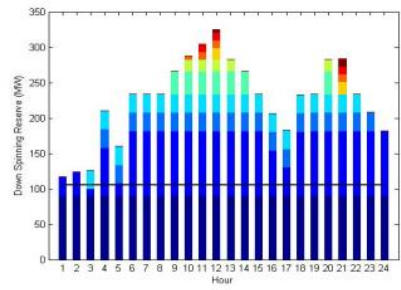

(c2)
Fig. 6. Scheduled system-wide SR for case study 3: (a1) Up SR of solution1, (b1) Up SR of solution2, (c1) Up SR of solution3 and (a2) Down SR of solution1, (b2) Down SR of solution2, (c2) Down SR of solution3.

It is also worth noting that a fast non-dominated sorting approach is adopted in this paper so as to reduce the computational complexity from $O\left(n o . n p^{3}\right)$ to $O(n o . n p \cdot \sqrt{n p})$, where no is the number of objectives and $n p$ is the population size [14]. This Pareto-optimal front is provided in 3.91 minutes. As can be seen from Fig. 4, generally, a lower OR corresponds to a higher operational cost, i.e., more up/down SR. According to the obtained Pareto-optimal front, a final solution can be selected by the system operator according to the practical requirements as well as engineering judgments. The optimal operational cost found by NSBSO algorithm with $20 \%$ WPPs is $\$ 528,923.16$. The OR is \%0.042. The scheduling of this case study as RCUC, is less risky than that of the deterministic UC in Case Study 2 due to the detailed modeling of the wind power and demand uncertainties.

The hourly wind curtailment levels for three mentioned solutions are shown in Table III. What should be underscored is that the wind curtailment level of solution1 (Best cost) is larger than the solution2 (Best compromise) and solution3 (Best OR), with the peak wind curtailment levels of 7 during periods of low demand and high wind power i.e. hours 1-7 and 20-24. It means that despite the fact that wind is free, there are periods of time when reaching to the lower OR dose not permit wind power generators to inject all available wind power. Fig. 5 and Fig. 6 illustrate power outputs of committed units and scheduled up/down SR for three mentioned solutions. As can be seen, these SR have the essential role in Pareto-optimal front especially on OR. Both up/down SR increase from solutions 1 to 3 as the OR decreases, suggesting that the value of the SR is determined by the OR. It can be seen from Fig. 6 that the number of on/off statuses or operation cycles is increased from solution1 to solution3, a characteristic that tends to increase the start-up cost and decrease the OR. Indeed, the expensive TUs 6 to 9 which are committed in satisfying peak demand of previous solution 1 and 2, need to be scheduled in solution 3 in order to decrease the OR and provide SR requirements in different hours. In addition, it is clear that the number of committed units in all hours of solution3 is higher than or equal to solution1 and 2 due to more SR requirements. Fig. 7 examines the impacts of different levels of WPP on the Pareto-optimal front. As the WPPs are increased, the operational cost and OR are decreased and increased, respectively. We now explore the effect of WPC on Paretooptimal front. Fig. 8 shows the Pareto-optimal front in both states of considering/neglecting WPC for the WPPs of $20 \%$. It is clear that the Pareto-optimal front provided in the first state dominates the Pareto-optimal front achieved in second one indicating the impacts of WPC on decreasing both start up and generation cost as well as OR, simultaneously.

\section{B. Second Test System: IEEE 118-bus with 54 units}

In this case, the IEEE 118-bus system with 54-units shown in [25] is selected. All the RCUC problem constraints (4a)(4e) are considered. Note, the worst-case contingency scenario as the $n-1$ security criterion is also deliberated. Firstly, the single objective optimization is applied to determine the extreme points of the trade-off surface.

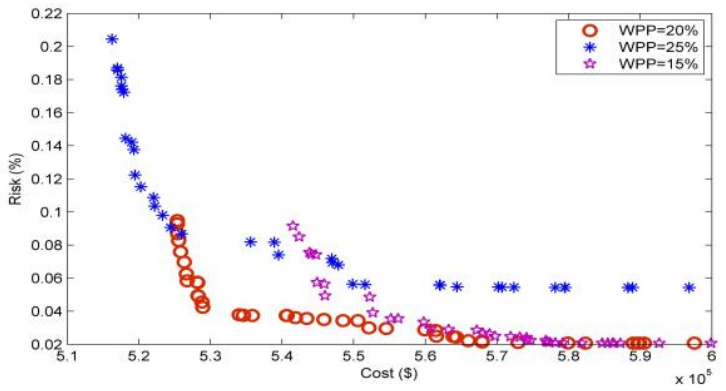

Fig. 7. Pareto-optimal front obtained by proposed NSBSO for different WPPs.

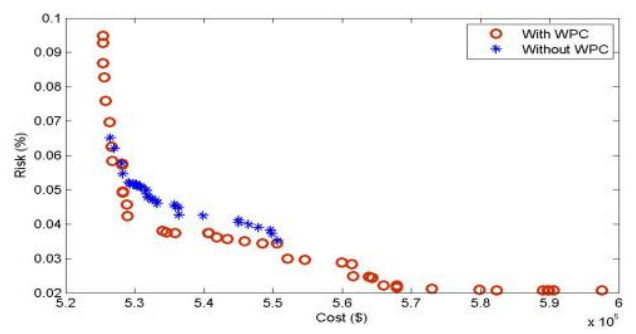

Fig. 8. Pareto-optimal front obtained by proposed NSBSO considering the WPC effect.

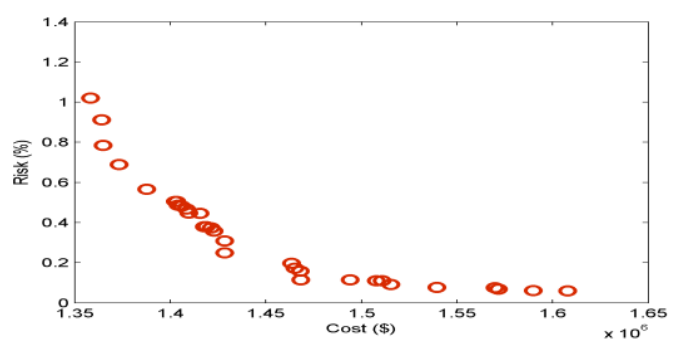

Fig. 9. Pareto-optimal front obtained by NSBSO for IEEE 118-bus with 54 units system. 
TABLE IV

NuMBER OF COMMITTED UNITS OF DiFFERENT SOLUTIONS OF PARETOOPTIMAL FRONT FOR SECOND SYSTEM

\begin{tabular}{ccccccccccccc}
\hline Hours & \multicolumn{10}{c}{$\mathbf{1 - 1 2}$} \\
\hline Solution1 & 15 & 15 & 19 & 15 & 15 & 19 & 16 & 19 & 22 & 22 & 23 & 25 \\
\hline Solution2 & 34 & 38 & 38 & 34 & 38 & 29 & 37 & 38 & 37 & 39 & 48 & 49 \\
\hline Solution3 & 46 & 38 & 43 & 41 & 45 & 39 & 40 & 45 & 45 & 39 & 42 & 49 \\
\hline Hours & & & & \multicolumn{1}{c}{$\mathbf{1 3 - 2 4}$} & & & & & \\
\hline Solution1 & 23 & 23 & 32 & 27 & 27 & 26 & 27 & 27 & 25 & 36 & 48 & 53 \\
\hline Solution2 & 43 & 43 & 43 & 44 & 49 & 42 & 45 & 49 & 51 & 50 & 47 & 42 \\
\hline Solution3 & 49 & 48 & 46 & 41 & 48 & 46 & 48 & 46 & 50 & 43 & 44 & 38 \\
\hline
\end{tabular}

The optimal operational cost and its related OR are found to be $\$ 1,358,263$ and $1.02 \%$, respectively. The optimal OR and its related operational cost are $0.06 \%$ and $\$ 1,607,699$, respectively. The required simulation time for this case is around 8.88 minutes. Then, the proposed solution method is applied to solve the bi-objective optimization problem under 20\% WPPs. Fig. 9 shows the provided Pareto-optimal front in 15.61 minutes. The operational cost and risk for the best compromise solution are $\$ 1,428,413$ and $0.25 \%$, respectively. In this case study, the number of committed units corresponding to solution1, solution2 and solution3 are tabulated in Table IV. It is evident that the numbers of TUs which are committed during the whole day are totally increased from solution1 to solution2 i.e. 599 to 1,007 and from solution2 to solution 3 i.e. 1,007 to 1,059 . This is due to the OR reduction requirement. In addition, based on the output of the optimization process and $n-1$ security restriction, the largest unit is not turned on during the studying period.

\section{CONCLUSION}

In this paper, an efficient framework to solve the projected RCUC addressing demand and wind power uncertainties in a compact way is proposed. The risks of wind power curtailment and probable unit outages are modeled as the operational risk objective function besides the operational cost. The new biobjective NSBSO solution methodology is suggested and utilized to handle the RCUC problem. The heuristic strategies proposed in this paper have the potential to handle all the constraints especially the up/down ramp rates. Moreover, the new redefining of binary on/off variables by operation cycles is also proposed. The illustrative example and case studies show how the system operator can select a best compromise solution from the Pareto-optimal front which satisfies both objective functions. The model can be solved with high efficiency and can be applied on larger power systems.

The proposed framework can be followed in the future work by addressing an efficient market mechanism under correlations between input random variables as well as demand response to improve generated and consumed schedules using the proposed RCUC architecture.

\section{REFERENCES}

[1]Midwest ISO, Energy and Operating Reserve Markets Business Practices Manual, BPM-002-r9.

[2]A. Trivedi, D. Srinivasan, K. Pal, C. Saha, and T. Reindl, "Enhanced multiobjective evolutionary algorithm based on decomposition for solving the unit commitment problem," IEEE Trans. Ind. Informat., vol. 11, no. 6, pp. 1346-1357, Nov. 2015.

[3]H. Khorramdel, J. Aghaei, B. Khorramdel, and P. Siano, "Optimal battery sizing in microgrids using probabilistic unit commitment," IEEE Trans. Ind. Informat., vol. 6, no. 2, pp. 834-843, Apr. 2016.
[4]Z. Asad, M. A. R. Chaudhry, and D. Kundur, "On the use of matroid theory for distributed cyber-physical-constrained generator scheduling in smart grid," IEEE Trans. Ind. Informat., vol. 62, no. 1, pp. 299-309, Jan. 2015.

[5]W. Lyzwa, M. Wierzbowski, and B. Olek, "MILP formulation for energy mix optimization," IEEE Trans. Ind. Informat., vol. 11, no. 5, pp. 11661178, Oct. 2015.

[6]A. Muzhikyan, A. M. Farid, and K. Youcef-Toumi, "An enterprise control assessment method for variable energy resource-induced power system imbalances-Part I: Methodology," IEEE Trans. Ind. Electronics, vol. 62, no. 4, pp. 2448-2458, Apr. 2015.

7]L. Soder, "Reserve margin planning in a wind-hydro-thermal power system," IEEE Trans. Power Syst., vol. 8, no. 2, pp. 564-571, May 1993.

[8]L. Guodong and K. Tomsovic, "Quantifying spinning reserve in systems with significant wind power penetration," IEEE Trans. Power Syst., vol. 27, no. 4, pp. 2385-2393, Nov. 2012.

[9]P. Xiong and P. Jirutitijaroen, "A stochastic optimization formulation of unit commitment with reliability constraints," IEEE Trans. Smart Grid, vol. 4, no. 4, pp. 2200-2208, Dec. 2013.

[10] J. Wang, M. Shahidehpour, and Z. Li, "Security-constrained unit commitment with volatile wind power generation," IEEE Trans. Power Syst., vol. 23, no. 3, pp. 1319-1327, Aug. 2008.

[11] N. Zhang, C. Kang, Q. Xia, Y. Ding, Y. Huang, R. Sun, J. Sun, and J. Bai, "A convex model of risk-based unit commitment for day-ahead market clearing considering wind power uncertainty," IEEE Trans. Power Syst., vol. 30, no. 3, pp. 1582-1592, May 2015.

[12] J. F. Restrepo and F. D. Galiana, "Assessing the yearly impact of wind power through a new hybrid deterministic/stochastic unit commitment," IEEE Trans. Power Syst., vol. 26, no. 1, pp. 401-410, Feb. 2011.

[13] I. G. Damousis, A. G. Bakirtzis, P. S. Dokopoulos, A solution to the unit-commitment problem using integer-coded genetic algorithm, IEEE Trans. Power Syst., vol. 19, no. 2, pp. 1165-1172, May 2004.

[14] X. Zhang, Y. Tian, R. Cheng, and Y. Jin, "An efficient approach to non-dominated sorting for evolutionary multi-objective optimization," IEEE Trans. Evol. Comput., vol. 19, no. 2, pp. 201-213, April 2015.

[15] P. Civicioglu, "Backtracking Search Optimization Algorithm for numerical optimization problems," Appl. Math. Comput., vol. 219, pp. 8121-8144, 2013.

[16] R. Azizipanah-Abarghooee, V. Terzija, F. Golestaneh and A. Roosta, "Multiobjective Dynamic optimal power flow considering fuzzy-based smart utilization of mobile electric vehicles," IEEE Trans. Ind. Informat., vol. 12, no. 2, pp. 503-514, April 2016.

[17] R. Azizipanah-Abarghooee, P. Dehghanian and V. Terzija, "Practical multi-area bi-objective environmental economic dispatch equipped with a hybrid gradient search method and improved Jaya algorithm," IET Gener. Transm. Distrib., pp. 1-17, In press, 2016.

[18] M. Zare, T. Niknam, R. Azizipanah-Abarghooee, A. Ostadi, "New stochastic bi-objective optimal cost and chance of operation management approach for smart micro-grid," IEEE Trans. Ind. Informat., vol. PP, no. 99, pp. 1-10, 2016

[19] R. Azizipanah-Abarghooee, F. Golestaneh, H. B. Gooi, J. Lin, F. Bavafa, and V. Terzija, "Corrective economic dispatch and operational cycles for probabilistic unit commitment with demand response and high wind power," Appl. Energy, vol. 182, pp. 634-651, 2016.

[20] S. A. Kazarlis, A. Bakirtzis, and V. Petridis, "A genetic algorithm solution to the unit commitment problem," IEEE Trans. Power Syst., vol. 11, no. 1, pp. 83-92, 1996.

[21] "Ontario power authority database." [Online]. Available: http://www.powerauthority.on.ca

[22] Fabbri, T. G. S. Roman, J. R. Abbad, and V. M. Quezada, "Assessment of the cost associated with wind generation prediction errors in a liberalized electricity market," IEEE Trans. Power Syst., vol. 20, no. 3, pp. 1440-1446, 2005.

[23] T. Niknam, F. Bavafa, and R. Azizipanah-Abarghooee, "New selfadaptive bat-inspired algorithm for unit commitment problem," IET Sci. Meas. Technol., vol. 8, no. 6, pp. 505-517, May 2014.

[24] S. Chakraborty, T. Ito, T. Senjyu, and A. Y. Saber, "Intelligent economic operation of smart-grid facilitating fuzzy advanced quantum evolutionary method," IEEE Trans. Sustain. Energy, vol. 4, no. 4, pp. $905-$ 916, 2013.

[25] The Electrical and Computer Engineering Department, Illinois Institute of Technology, Data. Available at: http://motor.ece.iit.edu/data/IEEE118bus_inf. 
Farhad Bavafa received the B.Sc. and M.Sc. degrees in control and power electrical engineering from Shiraz University of Technology, Shiraz, Iran in 2011 and 2013, respectively. His research interests include classical controller design techniques and power systems operation and control.

Taher Niknam (M'14) received the B.Sc. degree from Shiraz University, Shiraz, Iran, in 1998, and the M.Sc. and Ph.D. degrees from the Sharif University of Technology, Tehran, Iran, in 2000 and 2005, respectively, all in power electrical engineering. He is a Faculty Member with the Department of Electrical and Electronic Engineering, Shiraz University of Technology. He was awarded as the young investigator by Iranian IEEE Branch in 2014, and selected by the ministry of higher education as the distinguished professor in 2015. His research interests include power system restructuring, impacts of distributed generations on power systems, optimization methods, and evolutionary algorithms.

Rasoul Azizipanah-Abarghooee ( $S^{\prime}$, 15) received the B.Sc. and M.Sc. degrees in power electrical engineering from Isfahan University of Technology, Isfahan Iran, and Shiraz University of Technology, Shiraz, Iran in 2010 and 2012 respectively. He is also a Research Assistant at the School of Electrical and Electronic Engineering, University of Manchester, Manchester, U.K. His research focuses on smart grids, integration of renewable energy sources in the grid, power system dynamic analysis and operation, application of optimization techniques in market operation, practical forecasting and probabilistic programming.

Vladimir Terzija (M'95-SM'2000-F'2016) was born in Donji Baraci (former Yugoslavia). He received the Dipl-Ing., M.Sc., and Ph.D. degrees in electrical engineering from the University of Belgrade, Belgrade, Serbia, in 1988, 1993, and 1997, respectively.

$\mathrm{He}$ is the Engineering and Physical Science Research Council Chair Professor in Power System Engineering with the School of Electrical and Electronic Engineering, The University of Manchester, Manchester, U.K., where he has been since 2006. From 1997 to 1999, he was an Assistant Professor at the University of Belgrade, Belgrade, Serbia. From 2000 to 2006 , he was a senior specialist for switchgear and distribution automation with ABB AG Inc., Ratingen, Germany. His current research interests include smart grid application of intelligent methods to power system monitoring, control, and protection; wide-area monitoring, protection, and control; switchgear and fast transient processes; and digital signal processing applications in power systems.

Prof. Terzija is Editor in Chief of the International Journal of Electrical Power and Energy Systems, Alexander von Humboldt Fellow, as well as a DAAD and Taishan Scholar. 